\title{
Critical Exponents without the Epsilon Expansion
}

\author{
Mark Alford* \\ Laboratory of Nuclear Studies, \\ Cornell University, \\ Ithaca, NY 14853
}

March 21, 1994

CLNS 94/1279

hep-ph/9403324

\begin{abstract}
We argue that the sharp-cutoff Wilson renormalization group provides a powerful tool for the analysis of second-order and weakly first-order phase transitions. In particular, in a computation no harder than the calculation of the 1-loop effective potential, we show that the Wilson RG yields the fixed point couplings and critical exponents of 3-dimensional $O(N)$ scalar field theory, with results close to those obtained in high-order $\varepsilon$-expansion and large- $N$ calculations. We discuss the prospects for an even more precise computation.
\end{abstract}

\footnotetext{
*E-mail address: alford@hepth.cornell.edu
} 
Weakly first-order and second-order phase transitions have been important for some time in cosmology. Particular attention has been focused on the electroweak phase transition (EWPT), in an effort to determine whether it is first-order and thereby capable of generating the observed asymmetry between matter and antimatter in the universe [1]. However, progress has been complicated by infrared problems that invalidate the usual loop expansion [2]. In statistical mechanics, the usual remedy is to use the $\varepsilon$-expansion [3, 圆, and this has been applied to the EWPT [5]. However, it has been argued that the $\varepsilon$-expansion may be unreliable for the EWPT [6], since there may be a fixed point in three dimensions that is not visible in an expansion around four dimensions. In this letter we will show how a second-order phase transition can be analyzed by the "Wilson" or "exact" renormalization group (RG) [4, \$ directly in three dimensions, without recourse to the $\varepsilon$-expansion. It appears straightforward to extend this method to first-order phase transitions [14], which would enable us to determine the order and the dynamics of the EWPT and other cosmological phase transitions.

There has recently been a resurgence of interest in the Wilson RG ([9]-[14]). An important contribution was made by Tetradis and Wetterich [15], who used the smooth-cutoff Wilson RG to calculate critical exponents of a second-order phase transition, and found impressive agreement with the traditional methods mentioned above. Unfortunately, the use of the smooth cutoff greatly complicates the computation, and numerical methods are required to determine the differential flow equations, which are then solved numerically to obtain the exponents. We will find, in contrast, that the sharp-cutoff Wilson RG yields the fixed point couplings and critical exponents in a simple and almost completely analytic form, requiring no more effort than the calculation of the one-loop effective potential. Even with a radically truncated action, the resultant values are within about $10 \%$ of those found by the traditional methods. In the appendix we discuss the problems that arise with both smooth and sharp cutoffs in a more precise calculation.

The Wilson RG follows the flow of the effective action (free energy) as degrees of freedom are integrated out in successively shrinking momentum shells. This corresponds to calculating the effective action with an infra-red (IR) cutoff $\Lambda$ for the loop corrections, and sending $\Lambda \rightarrow 0$. The effective action then flows with $\Lambda$, its couplings obeying differential equations that we will study below. For high temperature systems, we are interested in the flow for the 3 dimensional theory, and if there is a second-order phase transition we expect to find a fixed point that is IR-attractive in all coupling directions except one. This corresponds to the fact that one linear combination of the $U V$ couplings (i.e. the temperature) must be fine-tuned to a critical value if the theory is to flow into 
the fixed point in the IR. For temperatures close to the critical one, the effective action stays very close to the critical point for a large range of $\Lambda$, and this causes the singular dependence of many quantities on $T-T_{c}$.

We will consider the $O(N)$ scalar field theory in 3 dimensions. It will turn out that on the critical surface the mass-squared flows to zero from below, so the true minimum is at a nonzero field. By the $O(N)$ symmetry we can choose this to lie in the $\phi_{1}$ direction, so we shift the field $\phi_{i}=\Phi \delta_{i 1}+\varphi_{i}$, and the effective action for the shifted field $\varphi$ is

$$
\begin{aligned}
\mathcal{F} & =\frac{1}{2} Z \partial_{\mu} \varphi_{i} \partial^{\mu} \varphi_{i}+V(\rho) \\
\rho & \equiv \frac{1}{2} \phi^{i} \phi_{i}=\rho_{0}+\Phi \varphi_{1}+\frac{1}{2} \varphi_{i} \varphi_{i}
\end{aligned}
$$

This effective action is parameterized by the wavefunction renormalization $Z$, the position $\rho_{0}=\frac{1}{2} \Phi^{2}$ of the minimum of the potential, and the derivatives of $V$ at $\rho_{0}$, $V_{n}=\partial^{n} V / \partial \rho^{n}\left(\rho_{0}\right)$. By definition of $\rho_{0}, V_{1}=0$.

The procedure for obtaining the Wilson $R G$ equations is simply to calculate the effective action in the usual way, but with an IR cutoff $\Lambda$ in the propagators. We then differentiate with respect to $\Lambda$ to obtain the flow of $Z, \rho_{0}$, and the $V_{n}$ with $\Lambda$ [8, 11, 12, 13].

The flow of $Z$ is given by the anomalous dimension $\eta$, which is discussed in the Appendix. For the sake of computational simplicity, we have assumed that $Z$ is a constant, so $\eta=0$. This is expected to be a good approximation, since $\varepsilon$ - and large- $N$ expansions indicate $\eta \lesssim 0.04$. The flow of the effective potential just corresponds to differentiating with respect to an IR cutoff $\Lambda$ in the momentum integral of the usual 1-loop formula [16], so we easily obtain the flow equation, first written down by Nicoll, Chang, and Stanley (Ref. [7], 1976):

$$
\left.\Lambda \frac{\partial V(\rho)}{\partial \Lambda}\right|_{\rho}=-\frac{K_{3}}{2} \Lambda^{3}\left[\ln \left(Z \Lambda^{2}+V^{\prime}(\rho)+2 \rho V^{\prime \prime}(\rho)\right)+(N-1) \ln \left(Z \Lambda^{2}+V^{\prime}(\rho)\right)\right]
$$

where $K_{3}=1 /\left(2 \pi^{2}\right)$. We emphasize that although this looks like a 1-loop result there are no higher loop terms: the two-loop contribution from a given shell goes as $(d \Lambda)^{2}$ and does not affect the $\Lambda$-derivative. The approximation in Eq. (2) is that it uses a truncated effective action, only taking account of the flow of the momentum-independent couplings (the effective potential). Including momentum-dependent vertices would clearly improve the accuracy of the results.

Since we expect the dimensionless couplings to flow to a fixed point, we now scale out the dimensions and $Z$-dependence, defining a dimensionless field and couplings:

$$
\bar{\rho}=\rho \frac{Z}{\Lambda}, \quad v(\bar{\rho})=\Lambda^{-3} V=v_{0}+v_{1}\left(\bar{\rho}-\bar{\rho}_{0}\right)+\frac{1}{2} v_{2}\left(\bar{\rho}-\bar{\rho}_{0}\right)^{2}+\cdots
$$


Substituting these into Eq. (2) we find the flow of the dimensionless couplings at constant $\bar{\rho}$. Remembering that $\bar{\rho}$ is also flowing, our final result is

$$
\Lambda \frac{d v}{d \Lambda}=\Lambda \frac{d \bar{\rho}_{0}}{d \Lambda} \frac{\partial v}{\partial \bar{\rho}}-3 v+\bar{\rho} \frac{\partial v}{\partial \bar{\rho}}-\frac{1}{2} K_{3}\left\{\ln \left(1+v^{\prime}+2 \bar{\rho} v^{\prime \prime}\right)+(N-1) \ln \left(1+v^{\prime}\right)\right\} .
$$

We determine the flow of $\bar{\rho}_{0}$ by noting that by definition of $\bar{\rho}_{0}, v_{1}=0$ for all $\Lambda$, so the flow equation for $v_{1}$ becomes a flow equation for $\bar{\rho}_{0}$ :

$$
\Lambda \frac{d \bar{\rho}_{0}}{d \Lambda}=-\frac{1}{v_{2}} \Lambda \frac{\partial v_{1}}{\partial \Lambda}=-\bar{\rho}_{0}+\frac{1}{2} K_{3}\left((N-1)+\frac{3+2 \bar{\rho}_{0} v_{3} / v_{2}}{1+2 \bar{\rho}_{0} v_{2}}\right)
$$

We now expand Eq. (4) in powers of $\left(\bar{\rho}-\bar{\rho}_{0}\right)$ to find the flow of $v_{2}, v_{3}$, etc.

$$
\begin{aligned}
\Lambda \frac{d v_{2}}{d \Lambda}= & v_{3} \Lambda \frac{d \bar{\rho}_{0}}{d \Lambda}-v_{2}+\bar{\rho}_{0} v_{3} \\
- & \frac{1}{2} K_{3}\left((N-1)\left(v_{3}-v_{2}^{2}\right)-\frac{\left(3 v_{2}+2 \bar{\rho}_{0} v_{3}\right)^{2}}{\left(1+2 \bar{\rho}_{0} v_{2}\right)^{2}}+\frac{5 v_{3}+2 \bar{\rho}_{0} v_{4}}{1+2 \bar{\rho}_{0} v_{2}}\right) \\
\Lambda \frac{d v_{3}}{d \Lambda}= & v_{4} \Lambda \frac{d \bar{\rho}_{0}}{d \Lambda}+\bar{\rho}_{0} v_{4} \\
- & \frac{1}{2} K_{3}\left((N-1)\left(v_{2}^{3}-3 v_{2} v_{3}+v_{4}\right)+\frac{2\left(3 v_{2}+2 \bar{\rho}_{0} v_{3}\right)^{3}}{\left(1+2 \bar{\rho}_{0} v_{2}\right)^{3}}\right. \\
& \left.\quad-3 \frac{\left(3 v_{2}+2 \bar{\rho}_{0} v_{3}\right)\left(5 v_{3}+2 \bar{\rho}_{0} v_{4}\right)}{\left(1+2 \bar{\rho}_{0} v_{2}\right)^{2}}+\frac{7 v_{4}+2 \bar{\rho}_{0} v_{5}}{\left(1+2 \bar{\rho}_{0} v_{2}\right)}\right)
\end{aligned}
$$

Expanding to arbitrary order to find the flow of $v_{n}$ is a matter of tedious but straightforward algebra.

To find the fixed point we set all flows to zero and solve Eqs. (5) and (6) for the fixed point couplings $\bar{\rho}_{0}^{*}, v_{2}^{*}, v_{3}^{*} \ldots v_{n}^{*}$. If our results are to be trustworthy then the fixed point couplings must settle down at constant values as we relax the truncation, i.e. as $n \rightarrow \infty$. Fortunately, finding the fixed point always involves solving two simultaneous equations, no matter what the value of $n$, since the $\bar{\rho}_{0}$ equation eliminates $v_{3}^{*}$ in terms of $\bar{\rho}_{0}^{*}$ and $v_{2}^{*}$, the $v_{2}$ equation eliminates $v_{4}^{*}$, and so on. We finally set $v_{n+1}^{*}$ and $v_{n+2}^{*}$ to zero, obtaining two simultaneous (polynomial) equations for $\bar{\rho}_{0}^{*}$ and $v_{2}^{*}$. Solving these is the only part of the calculation which we have to perform numerically. The results for the two scalar field case $(N=2)$ are shown in Table 1 as a function of $n$. (They were obtained using the symbolic manipulation program Mathematica to analytically expand the flow equations and numerically find $\bar{\rho}_{0}^{*}$ and $v_{2}^{*}$.) We see impressive convergence of the couplings and the critical exponent $\beta$ (calculated below) as the truncation of the potential is relaxed.

To calculate critical exponents, consider what happens when $T$ is not quite equal to $T_{c}$, so that the dimensionless couplings almost flow into the fixed point, but turn away at 


\begin{tabular}{|c|c|c|c|c|c|c|c|c|c|}
\hline$n$ & $\beta$ & $\bar{\rho}_{0}^{*}$ & $v_{2}^{*}$ & $v_{3}^{*}$ & $v_{4}^{*}$ & $v_{5}^{*}$ & $v_{6}^{*}$ & $v_{7}^{*}$ & $v_{8}^{*}$ \\
\hline 3 & 0.401 & 0.0716 & 9.70 & 92.2 & 0 & 0 & 0 & 0 & 0 \\
4 & 0.390 & 0.0692 & 10.9 & 106 & 303 & 0 & 0 & 0 & 0 \\
5 & 0.385 & 0.0686 & 11.1 & 107 & 316 & -4660 & 0 & 0 & 0 \\
6 & 0.384 & 0.0685 & 11.2 & 108 & 335 & -4710 & 58400 & 0 & 0 \\
7 & 0.384 & 0.0684 & 11.2 & 108 & 335 & -4720 & 58400 & -64700 & 0 \\
8 & 0.384 & 0.0685 & 11.2 & 108 & 333 & -4730 & 53700 & -98400 & $-37 \mathrm{M}$ \\
\hline
\end{tabular}

Table 1: The $N=2$ fixed point couplings and critical exponent $\beta$, calculated using $n$ evolution equations, i.e. including vertices up to $\phi^{2 n}$. Note the striking convergence with increasing $n$. $\left(M \equiv 10^{6}\right)$.

the last moment and explode exponentially away from it. From Eq. (5) we see that when $\bar{\rho}_{0}$ becomes of order 1 , at some $\Lambda=\Lambda_{f}$, the noncanonical scaling term (multiplied by $K_{3}$ ) becomes negligible, and from then on $\bar{\rho}_{0}$ grows canonically, so $\rho_{0}$ tends to a constant:

$$
\rho_{0} \sim \Lambda_{f}
$$

The critical exponent $\beta$, defined by $\rho_{0} \sim\left(T-T_{c}\right)^{2 \beta}$, can then be found by examining the flows in the neighborhood of the fixed point. Write the couplings as a vector $\mathbf{v}$, with components $v_{i}-v_{i}^{*}$ (let $v_{1}$ be $\bar{\rho}_{0}$ for this purpose); then the flows can be linearized:

$$
\Lambda \frac{d \mathbf{v}}{d \Lambda}=\mathbf{W} \cdot \mathbf{v}
$$

so that

$$
W_{i j}=\left[\frac{d}{d v_{j}} \Lambda \frac{d\left(v_{i}-v_{i}^{*}\right)}{d \Lambda}\right]_{v=v^{*}}
$$

We will find that $\mathbf{W}$ has one positive eigenvalue $w_{1}$, corresponding to the repulsive direction, with eigenvector $\mathbf{w}_{1}$. It follows that

$$
\mathbf{v}(\Lambda)=A\left(T-T_{c}\right) \Lambda^{-w_{1}} \mathbf{w}_{1}+\cdots
$$

The couplings leave the vicinity of the fixed point when the coefficient of $\mathbf{w}_{1}$ becomes of order 1 , so $A\left(T-T_{c}\right) \Lambda_{f}^{-w_{1}} \sim 1$. By Eq. (17), we otain the critical exponent $\beta$ :

$$
\rho_{0} \sim\left(T-T_{c}\right)^{1 / w_{1}}, \quad \text { so } \quad \beta=1 /\left(2 w_{1}\right)
$$




\begin{tabular}{|c|c|c|c|c|c|}
\hline $\mathrm{N}$ & $\beta$ & $\bar{\rho}_{0}^{*}$ & $v_{2}^{*}$ & $v_{3}^{*}$ & $v_{4}^{*}$ \\
\hline 1 & $0.345[0.327(2)]$ & 0.0471 & 13.5 & 177 & 955 \\
2 & $0.385[0.349(4)]$ & 0.0686 & 11.1 & 107 & 316 \\
3 & $0.413[0.368(4)]$ & 0.0917 & 9.14 & 66.7 & 134 \\
10 & $0.473[0.449]$ & 0.266 & 3.60 & 9.06 & 6.44 \\
20 & $0.487[0.477]$ & 0.518 & 1.90 & 2.43 & 0.910 \\
50 & $0.495[0.491]$ & 1.28 & 0.776 & 0.405 & 0.0626 \\
100 & $0.497[0.496]$ & 2.54 & 0.391 & 0.103 & 0.00801 \\
\hline
\end{tabular}

Table 2: The $O(N)$ fixed point couplings and critical exponents for various $N$. The values in square brackets are the current best estimates, based on $\varepsilon$-expansion to order $\varepsilon^{5}(N \leq 3)$ [4], or large $N$ to order $1 / N^{2}(N \geq 10)$ [17].

where $w_{1}$ is the largest eigenvalue of the linearized flow equations (9) at the fixed point. We can now calculate the fixed point couplings, and the critical exponent $\beta$ for any $N$. (Except for the anomalous dimension, the other exponents follow by scaling relations.) The results are given in Table 2 .

We see that there is striking agreement between the sharp-cutoff Wilson RG, and conventional $\varepsilon$-expansion and large- $N$ results, particularly so considering the crudity of our approximation, in which all momentum dependence in the effective action has been ignored. These results also agree closely with those obtained numerically by Tetradis and Wetterich [15] via the smooth-cutoff Wilson RG. It seems reasonable to expect that the inclusion of momentum-dependent vertices would enhance the accuracy of the results still further. However, as we show in the appendix, this is not a completely straightforward procedure. In particular, the smooth-cutoff calculation [15] of the anomalous dimension appears to depend on the profile of the cutoff, rendering its physical significance unclear.

For cosmological applications, it is necessary to formulate a gauge-invariant version of the momentum cutoff, in order to study gauge theories with weakly first-order phase transitions such as the electroweak phase transition. Progress in this direction has already been reported [18]. It would also be interesting to see if the sharp-cutoff Wilson RG could be applied with equal success to second order phase transitions in other statistical mechanical models, or to calculation of the effective potential in other field theories where perturbation theory breaks down in the IR, such as multiple scalar field theories with radiatively induced first-order phase transitions of the Coleman-Weinberg type [14]. 


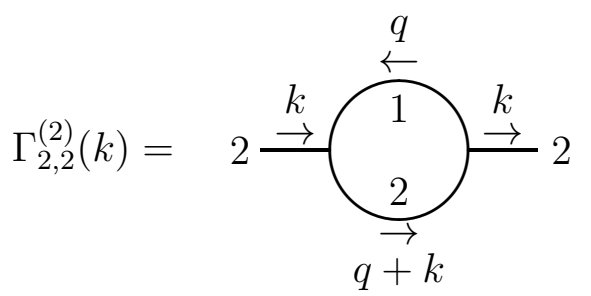

Figure 1: The diagram that contributes to the coefficient of $k^{2}$ in the 2-point 1PI Green's function, i.e. to the running of $Z$.

\section{Acknowledgments}

I would like to thank John March-Russell and Tim Klassen for discussions.

\section{Appendix A The anomalous dimension}

The anomalous dimension is given by the flow of the wavefunction renormalization $Z$, so it is convenient to express the effective action in terms of $\varphi_{i}$ rather than $\rho$ :

$$
V(\rho)=\frac{1}{2} V_{2}\left(\Phi^{2} \varphi_{1}^{2}+\Phi \varphi_{1} \varphi_{i} \varphi_{i}+\frac{1}{4} \varphi_{i} \varphi_{i} \varphi_{j} \varphi_{j}\right)+\cdots
$$

The first term is the mass of the radial mode, which comes into our flow formulae in its dimensionless form, as $2 \bar{\rho}_{0} v_{2}$. The next term provides a cubic interaction between the Goldstone and radial modes. This is the term that, via the graph shown in Fig. 1, will drive the cutoff-dependence of the wavefunction renormalization $Z$ of the Goldstone modes.

We calculate $Z=\frac{1}{2} \partial^{2} \Gamma_{2,2}^{(2)} / \partial k^{2}$ by evaluating this diagram in the usual way, but with an IR cutoff $\theta_{\varepsilon}\left(p^{2}-\Lambda^{2}\right)$ (which becomes a step function as $\varepsilon \rightarrow 0$ ) with every propagator. Differentiating with respect to $\Lambda$,

$$
\begin{gathered}
\eta_{g}=\frac{\Lambda}{Z} \frac{\partial Z}{\partial \Lambda}=\left(\Phi V_{2}\right)^{2} \frac{\Lambda}{Z} \frac{1}{2} \frac{\partial^{2}}{\partial k^{2}} \int \frac{d^{3} q}{(2 \pi)^{3}}\left\{\frac{\delta_{\varepsilon}(q-\Lambda)}{Z q^{2}+2 \rho_{0} V_{2}} \frac{\theta_{\varepsilon}\left((q+k)^{2}-\Lambda^{2}\right)}{Z(q+k)^{2}}\right. \\
\left.+\frac{\delta_{\varepsilon}(q-\Lambda)}{Z q^{2}} \frac{\theta_{\varepsilon}\left((q+k)^{2}-\Lambda^{2}\right)}{Z(q+k)^{2}+2 \rho_{0} V_{2}}\right\}
\end{gathered}
$$

This formula is to be evaluated in the limit $k \rightarrow 0$, and the sharp cutoff limit $\varepsilon \rightarrow 0$; but in which order? Clearly if we send $k \rightarrow 0$ first then the $k$-derivatives convert the $\theta_{\varepsilon}$-functions into $\delta_{\varepsilon}$-functions (and their derivatives) centered at $q=\Lambda$, and we obtain a result that is sensitively dependent on $\varepsilon$, the profile of the cutoff, and diverges as $\varepsilon \rightarrow 0$. Moreover, the expansion in $k$ is only valid for $k \lesssim \varepsilon$ [13]. This is not a problem with the 
sharp cutoff limit, as has been suggested [10, 11], since it is just as bad for the smooth cutoff to give profile-dependent results as it is for the sharp cutoff to give infinite ones. Rather, it implies that the order of limits is wrong. Physically, we want our effective action to be valid for momenta spanning as much of the range 0 to $\Lambda$ as possible. This is achieved by sending $\varepsilon \rightarrow 0$ first, and we then obtain $\varepsilon$-independent results for any $k \gg \varepsilon$, just as one would expect.

A more serious problem is that the resultant 2-point function contains a non-local $|k|$ term, as well as the expected $k^{2}$ term (see also Ref. [11]). This occurs because we have the same infrared cutoff on two different loop momenta, and only part of the loop is integrated out. This does not mean that the Greens functions, which are physically measurable, are non-local. The effective action with an IR cutoff is not a physically measurable quantity, and so it may indeed contain non-local terms [13]. It is not clear whether we should include a $|k|$ term in our effective action, with a separate flow equation for its coefficient, or whether we can amend the definition of the coarse-grained effective action in such a way as to eliminate non-local terms. This issue clearly deserves further study.

\section{References}

[1] A. Cohen, D. Kaplan, and A. Nelson, Annu. Rev. Nucl. Part. Sci. 43, 27 (1993).

[2] P. Arnold and O. Espinosa, Phys. Rev. D47, 3546 (1991), and references therein.

[3] K. Wilson and M. Fisher, Phys. Rev. Lett. 28, 240 (1978).

[4] J. Zinn-Justin, Quantum Field Theory and Critical Phenomena, Oxford University Press (1989).

[5] P. Arnold and L. Yaffe, Washington preprint UW/PT-93-24, hep-ph/9312221.

[6] J. March-Russell, Phys. Lett. B296, 364 (1992).

[7] K. Wilson, Phys. Rev. B4, 3174 and 3184 (1971);

K. Wilson and J. Kogut, Phys. Rep. 12C, 75 (1974);

F. Wegner and A. Houghton, Phys. Rev. A8, 401 (1973);

J. Nicoll, T. Chang, and H. Stanley, Phys. Rev. Lett. 33, 540 (1974), and Phys. Lett. 57A, 7 (1976);

K. Kawasaki, T. Imaeda, and J. Gunton, in Perspectives in Statistical Physics, (North-Holland, 1981), ed. H. Raveché. 
[8] J. Polchinski, Nucl. Phys. B231, 269 (1984).

[9] A. Hasenfratz and P. Hasenfratz, Nucl. Phys. B270, 685 (1986);

T. Hurd, Commun. Math. Phys. 124, 153 (1989);

G. Keller and C. Kopper, Phys. Lett. B273, 323 (1991);

T. Clark, B. Haeri, and S. Love, Nucl. Phys. B402,628 (1993).

[10] U. Ellwanger, Z. Phys C58, 619 (1993).

[11] C. Wetterich, Nucl. Phys. B352, 529 (1991) and Z. Phys. C57, 451 (1993).

[12] M. Bonini, M. D’Attanasio, G. Marchesini, Nucl. Phys. B409, 441 (1993).

[13] T. Morris, CERN preprint CERN-TH-6977-93, hep-ph/9308265.

[14] M. Alford and J. March-Russell, CLNS 93/1244, hep-ph/9308364.

[15] N. Tetradis and C. Wetterich, preprint DESY 93-094 (1993), hep-ph/9308214.

[16] S. Coleman, Aspects of Symmetry (Cambridge University Press, 1985), Ch. 5, Sect. 3.5.

[17] I. Kondor and T. Temesvari, J. Phys. Lett. (Paris) 39, L99 (1978).

[18] M. Reuter and C. Wetterich, DESY preprint 93-006;

M. Bonini, M. D'Attanasio, G. Marchesini, University of Parma preprint UPRF 93-388, hep-th/9312114. 